\title{
The logical bases of contradictory Christology: comments on The Contradictory Christ, Ch. 2
}

\author{
LuIs EstradA-GONZÁLEz ${ }^{1,2}$ \\ http://orcid.org/0000-0002-1466-0240 \\ ${ }^{1}$ Institute for Philosophical Research, UNAM \\ Mexico City \\ Mexico \\ ${ }^{2}$ Department of Logic, Nicolaus Copernicus University \\ Toruń \\ Poland \\ loisayaxsegrob@gmail.com
}

\author{
Article Info \\ CDD: 160.1 \\ Received: 15.06.2021; Accepted: 19.08.2021 \\ https://doi.org/10.1590/0100-6045.2021.V44N4.LG \\ Keywords: \\ Conditional \\ Detachment \\ Contraposition
}

${ }^{*}$ This paper was written during the COVID-19 crisis, under the financial support of the Notre Dame International-Mexico Faculty Grant Program project "The scope and limits of nondetachable conditionals", the PAPIIT project IN403719 and the Uniwersytet Mikolaja Kopernika w Toruniu (Excellence Initiative - Research University Program, Application 437). I presented a previous version during the online symposium "Discussing The Contradictory Christ"; I want to thank Jc Beall who is himself a demon and an archbishop - and the audience for the discussion. 
Abstract: Beall has given more or less convincing arguments to the effect that neither classical logic, nor $\mathbf{K}_{3}$, nor $\mathbf{L P}$, nor S3 can play the role he expects from logic: to be the basement theory for all true theories, including true theology. However, he has not considered all the pertinent competitors, and he has not given any reassurance that he has not gone too low in the hierarchy of logics to find his desired "universal closure of all true theories". In this paper, I put forward those additional arguments to show the superiority of FDE with respect to logics that include a detachable conditional but that are very much like FDE otherwise. I also discuss the problem that theological consequence might not contrapose even if theological consequence is supposed to extend FDE consequence and the latter does contrapose.

\section{Introduction}

In The Contradictory Christ ( [8] ${ }^{1}$, Jc Beall develops the basics of a contradictory Christology, that is, a theory about Christ that contains contradictions, such as "Christ is mutable and not mutable". To have such a theory, he needs a theory of logical consequence where contradictions do not imply any other formula whatsoever, and Chapter 2 of The Contradictory Christ is devoted to present that theory of logical consequence.

Beall says that, "for present purposes, [he] shall only assume that FDE is the correct account of logic (...)." (p. 27f.) But he does more than that. He at least sketches some arguments for that assumption, arguments that draw upon previous work by Beall on the topic, such as [5], [6], [7], [9]. Although he has dispatched other options - classical logic, $\mathbf{K}_{3}, \mathbf{L P}, \mathbf{S 3}^{2}$ more or less satisfactorily, this has not been done for all

\footnotetext{
${ }^{1}$ All quotations mentioning only page numbers are from this text.

${ }^{2}$ The intersection of $\mathbf{K}_{3}$ and $\mathbf{L P}$; see [14].
} 
the pertinent competitors. The feeling that he might have gone too low in the hierarchy of logics to find his desired "universal closure of all true theories" remains. I think this feeling must and can be put aside by showing the superiority of FDE over logics which are pretty close to it but that, unlike FDE, include a detachable conditional. That would make Beall's case stronger.

In this paper, I put forward those additional arguments, but let me be clear on my aim. Unlike Beall, I am not a supporter of the idea that FDE is "The One True Logic". Just as Beall thinks that logics stronger than FDE get more validities for extra-logical reasons, I think that there is still a lot of extra-logicality in FDE: logic alone should not validate anything. ${ }^{3}$ Nonetheless, I can join Beall in his quest of showing that no logic stronger than FDE is The One True Logic. But for that, the arguments for FDE must be refined and extended to deal with some obvious - and also with some not that obvious - competitors. This paper is devoted to that. My argument for going even weaker than FDE must be left for another occasion.

The plan of the paper is as follows. In Section 2, I give a quick reconstruction of Beall's view of logic, which involves giving FDE a distinguished status, very close to The One True Logic. In Section 3 I problematize such special status of FDE by its lack of a conditional that reflects entailment in the object-language. In particular, I present a couple of logics that seem to have all the nice features of FDE plus better conditionals. In Section 4, I discuss whether one can tip the scale in favor of FDE through the closeness of its evaluations conditions for the connectives to those of classical logic. Finally, in Section 5 I discuss the problem that theological consequence might not contrapose

\footnotetext{
${ }^{3}$ See for example the debate around [18] and [19], like [10], [11] and the now prehistoric [15] and [12].
} 
even if theological consequence is supposed to extend FDE consequence and the latter does contrapose.

\section{Beall's view of logic}

Why a reflection on logic is needed in the development of Christian theology? Here is Beall:

Christian theology is a theory of God, just as macro physics is a theory of the macrophysical world and just as mathematical theories are theories of their respective mathematical phenomena (numbers, categories, sets, whathaveyou). (...) [New paragraph] When theorists aim to construct a true theory, they aim to construct as complete a theory as possible. In particular, the resulting theory should not only contain the initial thrown-in truths (e.g., that God is triune, that Christ has two natures, etc.); the theory should also contain whatever follows from the truths in the theory; it should contain all of the consequences of the theory's claims. (p. 22)

And then he adds:

Without a consequence (closure) relation our theories remain inadequate; they fail to contain truths that are entailed by the given set of truths. Inasmuch as theorists, and theologians in particular, aim to give as complete a theory of the target phenomenon as possible, the reliance on a consequence relation for our theory is required. (p. 24)

But then he presents his own particular understanding of what logic is: 
Logic is a very special consequence (entailment, closure) relation. Logic is the common core of all (closed) theories; it is at the bottom of all of the (extra-logical, theoryspecific) consequence relations of our true theories. (...)

And, to say it briefly, logic can be that sort of theory because both its linguistic narrowness - it deals only with the so-called "logical vocabulary", vocabulary shared by all true theories-, and its semantic wideness -it considers all possible ways to interpret the vocabulary-.

As I mentioned in the Introduction, Beall thinks that FDE is the logic that has all those characteristics. My presentation of this logic will differ from Beall's but, hopefully, not substantially. Crucially, I do not consider Beall's nullation - an identity connective $\tau$ such that $\tau(A)$ has the same evaluations as $A-$, but consider the conditional separately, although it is definable through negation and disjunction. Also, I limit myself to the zero-order case.

FDE is a logic that can be presented as the result of evaluating formulas and arguments, built in the usual way from a countable set of propositional variables and the connectives $\{\sim, \wedge, \vee, \rightarrow\}$, according to the following assignments, where $A$ and $B$ stand for any formula:

- $\sim A$ is true iff $A$ is false; $\sim A$ is false iff $A$ is true

- $A \wedge B$ is true iff $A$ is true and $B$ is true; $A \wedge B$ is false iff $A$ is false or $B$ is false

- $A \vee B$ is true iff $A$ is true or $B$ is true; $A \vee B$ is false iff $A$ is false and $B$ is false

- $A \rightarrow B$ is true iff $A$ is false or $B$ is true; $A \rightarrow B$ is false iff $A$ is true and $B$ is false 
To achieve full generality with respect to the relations between formulas and truth values, the predications "is true" and "is false" should not be understood functionally, that is, being true does not imply not being false - nor vice versa-, and being false does not imply not being true - nor vice versa-. Said otherwise, a formula can be related to the truth values, via an assignment $\sigma$, in one of the following four ways:

- $A$ is true but not false, represented ' $1 \in \sigma(A)$ and $0 \notin \sigma(A)$ '; more briefly, $\sigma(A)=\{1\}$

- $A$ is true but also false, represented ' $1 \in \sigma(A)$ and $0 \in \sigma(A)^{\prime}$; more briefly, $\sigma(A)=\{1,0\}$

- $A$ is neither true nor false, represented ' $1 \notin \sigma(A)$ and $0 \notin \sigma(A)^{\prime}$; more briefly, $\sigma(A)=\{\}$

- $A$ is false but not true, represented ' $0 \in \sigma(A)$ and $1 \notin \sigma(A)^{\prime}$; more briefly, $\sigma(A)=\{0\}$

where each claim of the forms $v_{i} \in(A), v_{j} \notin(A)$, with $v_{i}, v_{j} \in\{1,0\}$, is called a Dunn atom. That these are the evaluations is what Beall means, at least partially, when he says that

Logic [i.e. FDE] (...) [recognizes] the widest space of possibilities. It's not that 'anything goes' by logic's lights; but a vast array of otherwise very strange possibilities is recognized by logic. (pp. 26f $)^{4}$

With this in mind, the evaluation conditions above for connectives can be represented tabularly as follows:

\footnotetext{
${ }^{4}$ To be fair, Beall requires extending the picture to get a predicate version of FDE. That would require that ( $n$-tuples of) objects are related in four ways to extensions and anti-extensions of ( $n$-ary) predicates: they belong to the extension but not to the anti-extension; they belong to both the extension and to the anti-extension, and so on. This can be safely ignored for the present purposes.
} 


\begin{tabular}{c|cc|cccc}
$\sim A$ & $A$ & $A \wedge B$ & $\{1\}$ & $\{1,0\}$ & \{\} & $\{0\}$ \\
\hline$\{0\}$ & $\{1\}$ & $\{1\}$ & $\{1\}$ & $\{1,0\}$ & \{\} & $\{0\}$ \\
$\{1,0\}$ & $\{1,0\}$ & $\{1,0\}$ & $\{1,0\}$ & $\{1,0\}$ & $\{0\}$ & $\{0\}$ \\
\{\} & \{\} & \{\} & \{\} & $\{0\}$ & \{\} & $\{0\}$ \\
$\{1\}$ & $\{0\}$ & $\{0\}$ & $\{0\}$ & $\{0\}$ & $\{0\}$ & $\{0\}$ \\
& $A \vee B$ & $\{1\}$ & $\{1,0\}$ & \{\} & $\{0\}$ & \\
& $\{1\}$ & $\{1\}$ & $\{1\}$ & $\{1\}$ & $\{1\}$ & \\
& $\{1,0\}$ & $\{1\}$ & $\{1,0\}$ & $\{1\}$ & $\{1,0\}$ & \\
\{\} & $\{1\}$ & $\{1\}$ & \{\} & \{\} & \\
& $\{0\}$ & $\{1\}$ & $\{1,0\}$ & \{\} & $\{0\}$ & \\
& $A \rightarrow B$ & $\{1\}$ & $\{1,0\}$ & \{\} & $\{0\}$ & \\
\cline { 2 - 5 } & $\{1\}$ & $\{1\}$ & $\{1,0\}$ & \{\} & $\{0\}$ & \\
& $\{1,0\}$ & $\{1\}$ & $\{1,0\}$ & $\{1\}$ & $\{1,0\}$ & \\
\{\} & $\{1\}$ & $\{1\}$ & \{\} & \{\} & \\
& $\{0\}$ & $\{1\}$ & $\{1\}$ & $\{1\}$ & $\{1\}$ &
\end{tabular}

Note that $\{1\},\{0\},\{1,0\}$ and \{\} are not truth values, but assignments or evaluations; more precisely, collections of truth values. ${ }^{5}$ So, under this presentation, it would be rather wrong to call FDE 'a four-valued logic'.

Finally, let $\Gamma$ be a set of formulas of FDE. Let us say that and argument is valid in FDE $\Gamma \vDash_{\mathbf{F D E}} A$, if and only if, for every evaluation $\sigma, 1 \in \sigma(A)$ if $1 \in$ $\sigma(B)$ for every $B \in \Gamma$. An argument is invalid in FDE if an only if there is an evaluation in which the premises are true, i.e. $1 \in \sigma(B)$ for every $B \in \Gamma$, but the conclusion is not, i.e. $1 \notin \sigma(A){ }^{6}$ This is what he means when he says

Logical entailment, like any entailment relation, is a relation of necessary truth preservation over relevant possibilities - in this

\footnotetext{
${ }^{5}$ One might say that they are generalized truth values. (See for example [20].) Let that be. It is still the case that they are not truth values like 1 and 0 .

${ }^{6}$ For simplicity, these definitions will be adapted for the other logics in this paper, just with the respective changes in language.
} 
case, the so-called logical possibilities (i.e., the possibilities recognized by logic). The recipe for logical entailment is absence of counterexample: (...) A counterexample is a possibility in which everything in $X$ [the premises in an argument] is true but $p$ [the conclusion] untrue. (p. 26)

According to Beall,

There are at least three salient virtues of this account of logic.

The first virtue is that the familiar truth and falsity conditions are maintained, as above. [Above: "What is important to emphasize $(. .$.$) is that these truth and fal-$ sity conditions are precisely the same conditions used in the standard classical-logic account. "Emphasis in the original.](...)

A second virtue is that the resulting logic is clearly topic-neutral by not taking a stand on whether gappy or glutty atomic sentences are ruled out.

A third virtue is most relevant to the principal project of a contradictory christology: namely, that logic does not force unique, strange phenomena into the cramped confines of classical-logic possibilities. (p. 35)

I will not deny that FDE has these virtues. What I will challenge is whether FDE possess them to the degree that Beall thinks it has them. More precisely, I will show that there is at least one more logic, deductively stronger than FDE, that has these virtues, and that if one wants to claim that FDE has a special status vi-a-vis that other logic, then more elaborated arguments, not yet given by Beall, will be needed. 


\section{The conditional}

Note that the conditional $A \rightarrow B$ in FDE is extensional as it is definable as $\sim A \vee B$-i.e. it is true iff the antecedent is false or the consequent is true-. It does not satisfy either of the following

- Identity: $\vDash A \rightarrow A$

- Detachment: $A, A \rightarrow B \vDash B$

- Deduction property: $A_{1}, \ldots A_{n} \vDash B$ iff $A_{1}, \ldots A_{n-1} \vDash$ $A_{n} \rightarrow B$

which are, for many, conditions sine qua non of conditionals. ${ }^{7}$ Thus, for example, Priest claims boldly that "FDE has no conditional operator" [17, p. 163].

I disagree, and I also disagree with people who claims something stronger, namely, that there must be conditionals in a logic. Nonetheless, they are a majority and the onus of the proof is on us. For simplicity, let me focus on an interlocutor in the middle, one that might accept that logic needs no conditional connective in the language, but that, pending further arguments, finds it preferable to have one, and one that satisfies the above properties above. They must be the kind of person that, when given the good arguments, accept that, according to certain criteria, FDE is better than any stronger logic, without then objecting that FDE is not a logic because it does not have a conditional. $^{8}$

${ }^{7}$ Countermodels: for the first, put $\sigma(A)=\{\}$; for the second, put $\sigma(A)=\{1,0\}$; for the third one, note that $A \vDash_{\text {FDE }} A$ but $\#_{\text {FDE }} A \rightarrow A$.

${ }^{8}$ I will leave aside the question whether the extensional conditional in FDE is a conditional or not; I will simply assume it is. One can construct some arguments from Beall's [3] or [4] to that effect. The gist would be that it has the evaluation conditions of a conditional, and that it validates certain restricted forms of the patterns demanded, which would be enough to consider it a conditional. See [13] for similar arguments. 
Consider Sylvan's suggestions of strengthening FDE into arrow form with a suitable conditional $\rightarrow_{\varphi}$, that is, changing every valid argument of the form $A \vDash_{\text {FDE }} B$ into one of the form $\vDash_{\mathbf{F D E}_{\rightarrow \varphi}} A \rightarrow_{\varphi} B .^{9}$ The result is depicted as follows:

\section{FDE}

$A \vDash_{\mathrm{FDE}} A$

$(A \wedge B) \vDash_{\mathrm{FDE}} A$

$(A \wedge B) \vDash_{\text {FDE }} B$

$A \vDash_{\mathrm{FDE}}(A \vee B)$

$B \vDash_{\mathrm{FDE}}(A \vee B)$

$A \wedge(B \vee C) \vDash_{\mathrm{FDE}}(A \wedge B) \vee C$

$\sim A \vDash_{\mathrm{FDE}} A$

$A \vDash_{\mathrm{FDE}} \sim A$

If $A \vDash_{\mathrm{FDE}} B, B \vDash_{\mathrm{FDE}} C$ then $A \vDash_{\mathrm{FDE}} C$

If $A \vDash_{\mathrm{FDE}} B, A \vDash_{\mathrm{FDE}} C$ then $A \vDash_{\mathrm{FDE}}(B \wedge C)$

If $A \vDash_{\mathrm{FDE}} C, B \vDash_{\mathrm{FDE}} C$ then $(A \vee B) \vDash_{\mathrm{FDE}} C$

If $A \vDash_{\mathrm{FDE}} \sim B$ then $B \vDash_{\mathrm{FDE}} \sim A$

\section{$\mathrm{FDE}_{\rightarrow \varphi}$}

$\mathrm{F}_{\mathrm{FDE}_{\rightarrow \varphi}} A \rightarrow \rightarrow_{\varphi} A$

${F_{\mathrm{FDE}_{\rightarrow \varphi}}}(A \wedge B) \rightarrow_{\varphi} A$

$\vDash_{\mathrm{FDE}_{\rightarrow \varphi}}(A \wedge B) \rightarrow_{\varphi} B$

$\vDash_{\mathrm{FDE}_{\rightarrow \varphi}} A \rightarrow \varphi(A \vee B)$

${₹_{\mathrm{FDE}_{\rightarrow \varphi}}} B \rightarrow \varphi(A \vee B)$

${F_{\mathrm{FDE}_{\rightarrow \varphi}}} A \wedge(B \vee C) \rightarrow \varphi(A \wedge B) \vee C$

$\mathrm{F}_{\mathrm{FDE}_{\rightarrow \varphi} \sim \sim A \rightarrow \varphi} A$

$\mathrm{F}_{\mathrm{FDE}_{\rightarrow \varphi}} A \rightarrow \varphi^{\sim \sim A}$

$A \rightarrow{ }_{\varphi} B, B \rightarrow{ }_{\varphi} C{\mathrm{~F}_{\mathrm{FDE}_{\rightarrow \varphi}}} A \rightarrow{ }_{\varphi} C$

$A \rightarrow{ }_{\varphi} B, A \rightarrow{ }_{\varphi} C \mathrm{~F}_{\mathrm{FDE}_{\rightarrow \varphi}} A \rightarrow_{\varphi}(B \wedge C)$

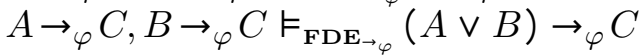

$A \rightarrow{ }_{\varphi} B \vDash_{\mathrm{FDE}_{\rightarrow \varphi}} \sim B \rightarrow_{\varphi} \sim A$

What does it take to have $\mathbf{F D E}_{\rightarrow_{\varphi}}$ ? It is FDE plus the following conditional:

${ }^{9}$ There are many places where he suggests this, but one particularly close to what I am discussing here is [21]. 


\begin{tabular}{c|cccc}
$A \rightarrow_{\varphi} B$ & $\{1\}$ & $\{1,0\}$ & \{\} & $\{0\}$ \\
\hline$\{1\}$ & $\{1\}$ & $\{0\}$ & $\{0\}$ & $\{0\}$ \\
$\{1,0\}$ & $\{1\}$ & $\{1\}$ & $\{0\}$ & $\{0\}$ \\
\{\} & $\{1\}$ & $\{0\}$ & $\{1\}$ & $\{0\}$ \\
$\{0\}$ & $\{1\}$ & $\{1\}$ & $\{1\}$ & $\{1\}$
\end{tabular}

whose evaluation conditions are as follows:

- $1 \in \sigma\left(A \rightarrow_{\varphi} B\right)$ iff (1) $0 \in \sigma(A)$ and $1 \notin \sigma(A)$, or (2) $1 \in \sigma(B)$ and $0 \notin \sigma(B)$, or $(3) 1 \in \sigma(A)$ iff $1 \in \sigma(B)$ and $0 \in \sigma(A)$ iff $0 \in \sigma(B)$

- $0 \in \sigma\left(A \rightarrow{ }_{\varphi} B\right)$ iff $(1) 1 \in \sigma(A), 0 \notin \sigma(A)$ and $0 \in \sigma(B)$, or $(2) 1 \in \sigma(A)$, and $1 \notin \sigma(B)$, or $(3)$ $1 \notin \sigma(A), 0 \notin \sigma(A)$ and $0 \in \sigma(B)$

In vernacular: this conditional is true iff either the antecedent is just false, or the consequent is just true, or both antecedent and consequent have the same evaluations. On the other hand, this conditional is false iff the antecedent is just true and the consequent is false, or the antecedent is true and the consequent is not, or the antecedent is neither true nor false but the consequent is false.

(Sylvan calls this conditional 'Philonian'. Although the reasons are not clear to me, I will follow him in this, especially to not always referring to it as ' $\rightarrow \varphi$ '. Note that if arrows are not allowed neither in antecedents nor in consequents, FDE $_{\rightarrow_{\varphi}}$ would simply become Anderson and Belnap's logic of tautological entailment, $\mathbf{E}_{f d e}$; see [1, Ch. 15]. I keep the name 'FDE $\rightarrow_{\varphi}$ ', - and the somewhat redundant axiomatization - to stress that this need not be first-order entailment i.e. there can be nested arrows - and because it makes clear an intuitive construction of the logic: one adds the Philonian arrow to FDE.)

FDE $_{\rightarrow_{\varphi}}$ seems to have the same three "salient virtues" as FDE: the familiar truth and falsity conditions are maintained; the resulting logic is clearly topic-neutral 
by not taking a stand on whether gappy or glutty atomic sentences are ruled out; it does not force unique, strange phenomena into the cramped confines of classicallogic possibilities. I will discuss in more detail the first virtue in the next section; for now, let me say some words on the other two.

Some might reply that $\mathbf{F D E}_{\rightarrow_{\varphi}}$ does take a stand on whether gappy sentences or glutty sentences are ruled out, as the Philonian conditional receives only classically admissible interpretations. Such a reply forgets that the topic-neutrality was required for atomic sentences only. Moreover, the Philonian conditional is representing logical validity in the object language and, like it or not, even in FDE validity is exclusive and exhaustive, i.e. arguments are either valid or invalid, so it is not a surprise that this conditional is either true (only) or false (only). ${ }^{10}$

A similar objection may be raised against the third virtue: in getting only classically admissible interpretations, the Philonian conditional would "force unique, strange phenomena into the cramped confines of classicallogic possibilities". But that does not follow. First, remember that the "unique, strange phenomena" are phenomena like the incarnation, which, according to Beall, have the form $A \wedge \sim A$, and these are not untrue under all interpretations, as in classical logic. Moreover, conditional relations involving these phenomena are not evaluated as in classical logic, either. Neither $(A \wedge \sim A) \rightarrow_{\varphi} B$ nor $A \rightarrow_{\varphi}(B \vee \sim B)$ are valid in $\mathbf{F D E}_{\rightarrow}$. For a countermodel to the first one, consider $\sigma(A)=\{1,0\}$ and $1 \notin \sigma(B)$; for the second one, consider $1 \in \sigma(A)$ and $1 \notin \sigma(B)$. Thus, it does not seem that "unique, strange phenomena" are forced "into the

\footnotetext{
${ }^{10}$ I think this is a defect of FDE, as the logic is often accompanied by claims of treating truth and falsity on equal footing, but that policy is not extended to validity and invalidity. Nonetheless, I will not press the issue further here.
} 
cramped confines of classical-logic possibilities".

As Ia have said, I do not think that these objections are very telling. Nonetheless, there is a detachable conditional that does not receive only classically admissible interpretations and thus avoid these objections right from the start. Consider this material conditional, $A \rightarrow{ }_{m} B$, evaluated as follows:

$1 \in \sigma\left(A \rightarrow_{m} B\right)$ iff $1 \notin \sigma(A)$ or $1 \in \sigma(B)$

$0 \in \sigma\left(A \rightarrow_{m} B\right)$ iff $1 \in \sigma(A)$ and $0 \in \sigma(B)$

whose truth table is as follows:

\begin{tabular}{c|cccc}
$A \rightarrow_{m} B$ & $\{1\}$ & $\{1,0\}$ & \{\} & $\{0\}$ \\
\hline$\{1\}$ & $\{1\}$ & $\{1,0\}$ & \{\} & $\{0\}$ \\
$\{1,0\}$ & $\{1\}$ & $\{1,0\}$ & \{\} & $\{0\}$ \\
\{\} & $\{1\}$ & $\{1\}$ & $\{1\}$ & $\{1\}$ \\
$\{0\}$ & $\{1\}$ & $\{1\}$ & $\{1\}$ & $\{1\}$
\end{tabular}

It can be easily verified that Detachment, Identity and the Deduction Property formulated for this conditional are valid in $\mathbf{F D E}_{\rightarrow_{m}}$, the logic resulting from adding $\rightarrow_{m}$ to FDE. ${ }^{11}$ It can also be easily verified that the following are not valid in $\mathbf{F D E}_{\rightarrow_{m}}$ :

$(A \wedge \sim A) \rightarrow_{m} B$

$A \rightarrow_{m}(B \vee \sim B)$

That $\mathbf{F D E} \rightarrow_{m}$ is different from $\mathbf{F D E}_{\rightarrow_{\varphi}}$ can be easily checked by noting that

$A \rightarrow_{m}\left(B \rightarrow_{m} A\right)$

and

$A \rightarrow_{m}\left(B \rightarrow_{m}(A \wedge B)\right)$

are valid in $\mathbf{F D E}_{\rightarrow_{m}}$, but $A \rightarrow_{\varphi}\left(B \rightarrow_{\varphi} A\right)$ and $A \rightarrow_{\varphi}$ $\left(B \rightarrow_{\varphi}(A \wedge B)\right)$ are not in $\mathbf{F D E}_{\rightarrow_{\varphi}}$.

Again, $\mathbf{F D E}_{\rightarrow_{m}}$ seems to have the same three "salient virtues" as FDE: the familiar truth and falsity conditions are maintained; the resulting logic is clearly topic-neutral by not taking a stand on whether gappy or glutty atomic sentences are ruled out; it does not

\footnotetext{
${ }^{11}$ This connective has been studied in [2], for example, where many results about it can be found.
} 
force unique, strange phenomena into the cramped confines of classical-logic possibilities. Moreover, it cannot be argued that the latter virtues are not met because the conditional gets only classically admissible evaluations.

One could argue that even if $\mathbf{F D E}, \mathbf{F D E}_{\rightarrow_{\varphi}}$ and FDE $_{\rightarrow_{m}}$ share the three virtues above, only the first one is the universal closure of our true theories. This is notoriously difficult to assess, as we would need a theory of the non-theory-specific vocabulary of all of our true theories, and assuming that such a theory is FDE's is question-begging. There is at least one non-question-begging reply to be attempted, though. According to Beall, it is the business of extra-logical theories to determine what truths there are. Since $\mathbf{F D E}_{\rightarrow_{\varphi}}$ and $\mathbf{F D E}_{\rightarrow_{m}}$ contain truths, they would be extra-logical theories.

Let us examine what kinds of truths there are in FDE $_{\rightarrow_{\varphi}}$. There are implicative truths - i.e. of the form $A \rightarrow \varphi B$-, for example, all instances of the axioms schemas. There are also non-implicative truths, for example, of the form $A \vee\left(B \rightarrow_{\varphi} B\right)$ or $\sim\left(\left(A \rightarrow_{\varphi}\right.\right.$ $\left.A) \wedge \sim\left(A \rightarrow_{\varphi} A\right)\right)$. But note that these non-implicative truths are non-organic. A schema - or formula- in a $\operatorname{logic} \mathbf{L}$ is organic if and only if none of its sub-schemas - respectively, subformulas - is logically valid in $\mathbf{L}$. Otherwise, it is non-organic. In fact, all non-implicative truths in $\mathbf{F D E}_{\rightarrow_{\varphi}}$ are non-organic. ${ }^{12}$ (Note that classical logic is full of (non-implicative) organic validities: $\sim(p \wedge \sim p), q \vee \sim q$, and so on.) Moreover, the organic parts in those truths are insubstantial: these

\footnotetext{
${ }^{12}$ Sketch of the proof. Suppose that there is a non-implicative organic schema $S$ in $\mathbf{F D E}_{\rightarrow_{\varphi}}$. This means that there is no subschema in $S$ that is a truth. It cannot be made of just $\sim \wedge$ and $\vee$ as the evaluations for these are as in FDE and there are no truths in FDE. Then $S$ must have an implicative subschema with $\rightarrow_{\varphi}$ and, by the evaluation conditions of the connectives, it must be a truth itself.
} 
non-implicative truths are equivalent to the implicative truth they contain. Thus, in a sense, there are only implicative truths in $\mathbf{F D E}_{\rightarrow_{\varphi}}$, which are but objectlanguage correlates of the FDE validity claims.

$\mathbf{F D E}_{\rightarrow_{m}}$ does not survive equally well this criticism. The organic parts in some of its truths are substantial: there are non-implicative truths that are not equivalent to the implicative truth they contain. For example, $A \vee\left(B \rightarrow_{m} B\right)$. Thus, it cannot be argued that there are only implicative truths in $\mathbf{F D E}_{\rightarrow_{m}}$.

The Philonian and material arrows are certainly different from Beall's "Boolean quartet". Both its truth and falsity condition are more complicated than those of negation, conjunction and disjunction. That is not unlike other approaches to logic, where the conditional requires a separate, qualitatively special treatment think for example in those theories where all the connectives but the conditional are extensional. Whether a case for the defectiveness of such truth conditions can be made is the subject of the next section.

\section{The evaluation conditions}

Suppose that one were to argue that FDE's evaluation conditions are better than $\mathbf{F D E}_{\rightarrow_{\varphi}}$ 's and $\mathbf{F D E}_{\rightarrow_{m}}$ 's. Since the three logics coincide in the $\{\sim, \wedge, \vee\}$-fragment, the claim amounts to the superiority of the evaluation conditions for the extensional conditional over those for the Philonian and material conditionals.

Note that the original claim was that FDE "enjoys exactly the same truth and falsity conditions for the logical vocabulary as the classical account", that "the meanings of logical vocabulary are what they've always been". That was in fact one of the "salient virtues" of FDE. However, the sameness or otherwise of evaluation conditions could be not that favorable for the FDE picture, and ditto for the claims of betterness. 
What counts as "the same truth and falsity conditions for the logical vocabulary as the classical account"'? Compare

A conditional is true iff the antecedent is false or the consequent is true

and

A conditional is true iff the antecedent is untrue or the consequent is unfalse

What of them is "the (same as the) truth condition for the conditional in the classical account"? In classical logic, truth and unfalsity, on the one hand, and falsity and untruth, on the other, are exchangeable, so it seems that there are no reasons to prefer one above the other as the truth condition for the conditional in the classical account.

Or there is? Let me start with a platitude: a supporter of classical logic is interested in getting certain valid arguments - in particular, certain valid arguments with an empty set of premises, i.e. logical truths - . This is not independent of the formulation of the evaluation conditions, of course, but it is difficult that, when deciding between two evaluation conditions, a supporter of classical logic claims as theirs one that delivers less classically valid arguments than the other.

To make the discussion less abstract, consider these two well-known cases:

- $1 \in \sigma(\sim A)$ iff $0 \in \sigma(A)$ $0 \in \sigma(\sim A)$ iff $1 \in \sigma(A)$

- $1 \in \sigma\left(\sim_{b} A\right)$ iff $1 \notin \sigma(A)$ $0 \in \sigma\left(\sim_{b} A\right)$ iff $0 \notin \sigma(A)$

The latter are read: "Not- $A$ is true iff $A$ is not true, and Not- $A$ is false iff $A$ is not false". To simplify the forthcoming discussion, let me call the first pair of conditions 'De Morgan (evaluation) conditions (for nega- 
tion)' and the second 'Boolean (evaluation) conditions (for negation)'.

I claim that a supporter of classical logic would lean towards endorsing the Boolean conditions instead of the De Morgan ones, for the Boolean conditions validate the following:

$$
\begin{aligned}
& \vDash A \wedge \sim_{b} A \\
A, \sim_{b} A & \vDash B \\
A & \vDash B \vee \sim_{b} B
\end{aligned}
$$

and it allows defining another conditional, $A \rightarrow_{b} B={ }_{\text {def. }} \sim{ }_{b}$ $A \vee B$ that is detachable and validates $\left(A \wedge \sim_{b} A\right) \rightarrow_{b} B$ $A \rightarrow_{b}\left(B \vee \sim_{b} B\right)$ $A \rightarrow_{b}\left(B \rightarrow_{b} A\right)$

Given this, I would say that the supporter of classical logic would recognize the Boolean conditions, and not the De Morgan ones, as theirs.

Maybe the classical scruples are not a good companion here, and Beall misidentified what is good with the FDE evaluation conditions. Compare again a pair of evaluation conditions:

- $1 \in \sigma\left(A \rightarrow_{\varphi} B\right)$ iff $0 \in \sigma(A)$ or $1 \in \sigma(B)$

$0 \in \sigma\left(A \rightarrow_{\varphi} B\right)$ iff $1 \in \sigma(A)$ and $0 \in \sigma(B)$

- $1 \in \sigma\left(A \rightarrow_{\varphi} B\right)$ iff $(1) 0 \in \sigma(A)$ and $1 \notin \sigma(A)$, or (2) $1 \in \sigma(B)$ and $0 \notin \sigma(B)$, or $(3) 1 \in \sigma(A)$ iff $1 \in \sigma(B)$ and $0 \in \sigma(A)$ iff $0 \in \sigma(B)$

$0 \in \sigma\left(A \rightarrow{ }_{\varphi} B\right)$ iff (1) $1 \in \sigma(A), 0 \notin \sigma(A)$ and $0 \in \sigma(B)$, or $(2) 1 \in \sigma(A)$, and $1 \notin \sigma(B)$, or $(3)$ $1 \notin \sigma(A), 0 \notin \sigma(A)$ and $0 \in \sigma(B)$

The FDE conditions are not only simpler — at least by the length of the conditions-, they are also homogeneous, that is, all their Dunn atoms are positive: they are claims of truth (such as " $A$ is true") or falsity (such as " $B$ is false"). In contrast, the evaluation conditions 
for the Philonian conditional are not only more complex, but heterogeneous: they involve claims of truth, untruth, falsity and unfalsity. From simplicity and homogeneity considerations desirable in logic, the FDE conditions seem superior to those of $\mathbf{F D E}_{\rightarrow_{\varphi}} \cdot{ }^{13}$

Nonetheless, simplicity and homogeneity, even when combined with the space of possibilities allowed by FDE, do not take one out of classical logic by themselves. As I have said, the homogeneity in the evaluation conditions of FDE is positive, that is, its Dunn atoms are positive: they are claims of truth or falsity. But homogeneity can be also negative: the Dunn atoms can be all negative, claims of untruth or unfalsity. This would lead to the following negative conditions logic (NCL):

Either $1 \in \sigma(p)$ or $1 \notin \sigma(p)$, and either $0 \in \sigma(p)$ or $0 \notin \sigma(p)$

$1 \in \sigma(\sim A)$ iff $1 \notin \sigma(A)$

$0 \in \sigma(\sim A)$ iff $0 \notin \sigma(A)$

$1 \in \sigma(A \wedge B)$ iff $0 \notin \sigma(A)$ and $0 \notin \sigma(B)$

$0 \in \sigma(A \wedge B)$ iff either $1 \notin \sigma(A)$ or $1 \notin \sigma(B)$

$1 \in \sigma(A \vee B)$ iff either $0 \notin \sigma(A)$ or $0 \notin \sigma(B)$

$0 \in \sigma(A \vee B)$ iff $1 \notin \sigma(A)$ and $1 \notin \sigma(B)$

$1 \in \sigma(A \rightarrow B)$ iff $1 \notin \sigma(A)$ or $0 \notin \sigma(B)$

$0 \in \sigma(A \rightarrow B)$ iff $0 \notin \sigma(A)$ and $1 \notin \sigma(B)$

NCL's evaluation conditions are as simple and homogeneous as FDE's, but they are negative. As expected, Beall is not going to like the output, since NCL validates $(A \wedge \sim A) \rightarrow B$, which would not be very useful for Beall's Christology. (Although NCL is not classical logic: $A \rightarrow A$ is not valid; simply put $1 \in \sigma(A)$ and $0 \in \sigma(A)$.)

Thus, the good thing - for Beall's project - about FDE's evaluation condition is not their resemblance

\footnotetext{
${ }^{13}$ The evaluation conditions for the material conditional are simpler than those for the Philonian one, almost as simple as those for the extensional one. Still, they are heterogeneous.
} 
of the classical conditions, neither their simplicity and homogeneity, but that their evaluation conditions are simple and homogeneous in a way that do not yield too much classicality, and this is good - again, for Beall's project - because classicality comes with the neglect of many legitimate - maybe even true - theories.

But that still does not rule $\mathbf{F D E}_{\rightarrow_{\varphi}}$ out. An argument to the effect that the conditional should be equally - i.e. simply and homogeneously - analyzed as the other connectives is missing. In fact, the opposite may be the case if one aims at an object-language correlate of logical entailment. In that sense, the conditional is not like the other connectives. Beall might reply that that is precisely the extra-logical feature of $\mathbf{F D E}_{\rightarrow_{\varphi}}$ : it deals with a particular subject — viz. logical entailment-, instead of dealing with any domain whatsoever, like FDE. I can happily grant that, merely highlighting that the argument for FDE needed to be more sustained than what Beall attempted up to now, not only in The Contradictory Christ but in his other works arguing for FDE.

\section{Theological consequence and the individua- tion of FDE}

To finalize, let me ask a question about the individuation of FDE. Beall says that theological consequence does not contrapose, which is correct if there are true theological contradictions. As an illustration, suppose that $\gamma$ is one of such true theological contradictions. Then, for any sentence of the theory (in this case, true theology, $\Theta$ ),

$A \vdash_{\Theta} \gamma$

Suppose that $A$ is true only in the theory. Then the following is invalid in the theory:

$\sim \gamma \vdash_{\Theta} \sim A$

Hence, theological consequence does not contrapose. 
As Pawl [16] has rightly pointed out, this seems at odds with the facts that FDE-logical consequence contraposes and theological consequence extends FDElogical consequence, but according to Beall it is not. According to him, a theory extends logic if and only if, if $\Gamma \vdash A$ then $\Gamma \vdash_{\Theta} A$. This means, for him, that there are no claims of the form $A \vdash B$ that are valid in FDE but invalid in $\Theta$. Nonetheless, $\Theta$ and FDE can disagree when it comes to Contraposition, since it is not of the form $A \vdash B$, but of the form If $A \vdash B$ then $C \vdash D$.

But despite of Beall's efforts, it is odd that FDElogical consequence contraposes, that theological consequence extends FDE-logical consequence and that nonetheless theological consequence does not contrapose. To use a nowadays popular terminology, FDE consists not only of valid inferences - like $A \vdash A$ or $A \vdash A \vee B-$, but also of valid meta-inferences, such as Contraposition. But then how is it possible that $\Theta$ extends FDE without validating its meta-inferences too? Then either $\Theta$ does not extend FDE, or it does it but FDE is just a collection of valid inferences, not of meta-inferences as well.

I think there is no need for Beall to enter such a dilemma: one can describe theological consequence as FDE plus some background, extra-logical premises, that is, one can transform expressions like ' $A \vdash_{\Theta} B$ ' into expressions like ' $\Gamma, A \vdash_{\mathbf{F D E}} B$ '. But then it is no wonder that one loses Contraposition, as it is a wellknown fact that Contraposition with multiple premises fails in FDE, that is, $\Gamma, A \vdash_{\mathrm{FDE}} B$ might hold but $\Gamma, \sim B \vdash_{\mathrm{FDE}} \sim A$ not. $\Theta$ and FDE do not disagree with regards to metainferences after all. 


\section{Conclusions}

Beall has given more or less convincing arguments to the effect that neither classical logic, nor $\mathbf{K}_{3}$, nor $\mathbf{L P}$, nor $\mathbf{S 3}$ can play the role he expects from logic: to be the basement theory for all true theories. Nonetheless, he has not considered all the pertinent competitors, and he has not given any reassurance that he has not gone too low in the hierarchy of logics to find his desired "universal closure of all true theories".

In this paper, I put forward those additional arguments to show the superiority of FDE with respect to logics that include a detachable conditional but that are very much like FDE otherwise. Finally, I discussed the problem that theological consequence might not contrapose even if theological consequence is supposed to extend FDE consequence and the latter does contrapose. I hope to have shown that theological consequence does not contrapose because FDE does not contrapose either in the pertinent sense.

\section{References}

[1] Anderson, A. R., And Belnap, N. D. Entailment: The Logic of Relevance and Necessity, Vol. I. Princeton University Press, Princeton, 1975.

[2] Arieli, O., And Avron, A. Reasoning with logical bilattices. Journal of Logic, Language and Information 5, 1 (1996), 25-63.

[3] Beall, J. Multiple-conclusion LP and default classicality. Review of Symbolic Logic 4, 2 (2011), 326-336.

[4] Beall, J. Free of Detachment: Logic, rationality, and gluts. Noûs 49, 2 (2015), 410-423. 
[5] Beall, J. There is no logical negation: true, false, both and neither. Australasian Journal of Logic 14, 1 (2017), 1-29.

[6] Beall, J. The simple argument for subclassical logic. Philosophical Issues 28, 1 (2018), 30-54.

[7] Beall, J. FDE as The One True Logic. In New Essays on Belnap-Dunn Logic, H. Omori and H. Wansing, Eds. Springer, Cham, 2019.

[8] Beall, J. The Contradictory Christ. Oxford University Press, New York, 2021.

[9] Beall, J., And Camrud, C. A note on FDE "all the way up". Notre Dame Journal of Formal Logic 61, 2 (2020), 283-296.

[10] Cotnoir, A. J. Logical nihilism. In Pluralisms in Truth and Logic, J. Wyatt, N. J. L. L. Pedersen, and N. Kellen, Eds. Palgrave MacMillan, 2018, pp. 301-329.

[11] Dicher, B. Requiem for logical nihilism, or: Logical nihilism annihilated. Synthese (2020), 1-24.

[12] EstradA-GonzÁlez, L. On the meaning of connectives: Apropos of a non-necessitarianist challenge. Logica Universalis 5, 1 (2011), 115-126.

[13] EstradA-GonzÁlez, L., AND RAmÍrezCÁmARA, E. Non-conditional contracting connectives. In Mathematics, Logics and their Philosophies. Essays in Honor of Mohammad Ardeshir, S. Rahman, M. Mojtahedi, and M. S. Zarepour, Eds. 2020, pp. 349-364.

[14] Kremer, M. Kripke and the logic of truth. Journal of Philosophical Logic 17, 3 (1988), 225-278.

[15] Mortensen, C. Anything is possible. Erkenntnis 30, 3 (1989), 319-337. 
[16] Pawl, T. Explosive theology: A reply to Jc Beall's "Christ - a contradiction". Journal of Analytic Theology 7, 1 (2019), 440-451.

[17] Priest, G. An Introduction to Non-Classical Logics. From If to Is, second ed. Cambridge University Press, Cambridge, 2008.

[18] Russell, G. An introduction to logical nihilism. In Logic, Methodology and Philosophy of Science-Proceedings of the 15th International Congress (2017), College Publications, pp. 125135.

[19] Russell, G. Logical nihilism: Could there be no logic? Philosophical Issues 28, 1 (2018), 308-324.

[20] Shramko, Y., And Wansing, H. Truth and Falsehood: An Inquiry into Generalized Logical Values. Springer, Dordrecht, Heidelberg, London, New York, 2011.

[21] Sylvan, R. On interpreting truth tables and relevant truth table logic. Notre Dame Journal of Formal Logic 33, 2 (1992), 207-215.

\section{(cc) BY}

